\title{
Acute respiratory distress syndrome in mechanically ventilated patients with community-acquired pneumonia
}

\author{
Catia Cilloniz (1) ${ }^{1}$, Miquel Ferrer ${ }^{1}$, Adamanthia Liapikou² ${ }^{2}$, Carolina Garcia-Vidal ${ }^{3}$, \\ Albert Gabarrus ${ }^{1}$, Adrian Ceccato ${ }^{1}$, Jorge Puig de La Bellacasa ${ }^{4}$, \\ Francesco Blasi ${ }^{5}$ and Antoni Torres ${ }^{1}$
}

Affiliations: ${ }^{1}$ Dept of Pneumology, Institut Clinic de Respiratori, Hospital Clinic of Barcelona - Institut d'Investigacions Biomèdiques August Pi i Sunyer (IDIBAPS), University of Barcelona, Ciber de Enfermedades Respiratorias (Ciberes), Barcelona, Spain. ${ }^{2}$ Respiratory Dept, Sotiria Chest Diseases Hospital, Athens, Greece. ${ }^{3}$ Infectious Disease Dept, Hospital Clinic of Barcelona, Barcelona, Spain. ${ }^{4}$ Dept of Microbiology, Hospital Clinic of Barcelona, Barcelona, Spain. ${ }^{5}$ Dept of Pathophysiology and Transplantation, Università degli Studi di Milano, IRCCS Fondazione Cà Granda Ospedale Maggiore Policlinico, Milan, Italy.

Correspondence: Miquel Ferrer, Dept of Pneumology, Hospital Clinic of Barcelona Villarroel 170, Barcelona 08036, Spain. E-mail: miferreraclinic.ub.es

@ERSpublications

In mechanically ventilated patients with community-acquired pneumonia, ARDS based on the Berlin criteria was not related to aetiology or mortality http://ow.ly/BDMm30izoCN

Cite this article as: Cilloniz C, Ferrer M, Liapikou A, et al. Acute respiratory distress syndrome in mechanically ventilated patients with community-acquired pneumonia. Eur Respir J 2018; 51: 1702215 [https://doi.org/10.1183/13993003.02215-2017].

ABSTRACT Our aim was to assess the incidence, characteristics, aetiology, risk factors and mortality of acute respiratory distress syndrome (ARDS) in intensive care unit (ICU) patients with communityacquired pneumonia (CAP) using the Berlin definition.

We prospectively enrolled consecutive mechanically ventilated adult ICU patients with CAP over 20 years, and compared them with mechanically ventilated patients without ARDS. The main outcome was 30 -day mortality.

Among 5334 patients hospitalised with CAP, 930 (17\%) were admitted to the ICU and 432 required mechanical ventilation; $125(29 \%)$ cases met the Berlin ARDS criteria. ARDS was present in $2 \%$ of hospitalised patients and $13 \%$ of ICU patients. Based on the baseline arterial oxygen tension/inspiratory oxygen fraction ratio, 60 (48\%), 49 (40\%) and 15 (12\%) patients had mild, moderate and severe ARDS, respectively. Streptococcus pneumoniae was the most frequent pathogen, with no significant differences in aetiology between groups. Higher organ system dysfunction and previous antibiotic use were independent risk factors for ARDS in the multivariate analysis, while previous inhaled corticosteroids were independently associated with a lower risk. The 30-day mortality was similar between patients with and without ARDS ( $25 \%$ versus $30 \%, \mathrm{p}=0.25$ ), confirmed by propensity-adjusted multivariate analysis.

ARDS occurs as a complication of CAP in $29 \%$ of mechanically ventilated patients, but is not related to the aetiology or mortality. 


\section{Introduction}

Community-acquired pneumonia (CAP) is associated with increased morbidity, mortality and health costs $[1,2]$. The incidence of severe CAP requiring intensive care unit (ICU) admission is increasing, both because of septic shock and the need for invasive mechanical ventilation (IMV) [3]. Despite global efforts to improve outcomes, mortality remains high in severe CAP [4-6]. Streptococcus pneumoniae is the leading cause of CAP; it is the underlying aetiological agent in $22 \%$ of patients requiring ICU admission [7], and $\sim 30 \%$ of these patients develop pulmonary complications during their clinical courses [8].

Acute respiratory distress syndrome (ARDS) is a potential complication of severe CAP that is reported in $\sim 3 \%$ of patients hospitalised with pneumococcal CAP [9]. This condition is characterised by the rapid development of severe acute respiratory failure, and is associated with high morbidity and mortality despite advances in supportive care and ventilator management [10, 11]. However, there is limited information regarding the incidence of ARDS, associated pathogens, risk factors and specific outcomes in hospitalised patients with severe CAP, especially in the era of the current Berlin definition, according to which patients must be receiving positive-pressure ventilation [12]. Although overall survival from ARDS is improving $[12,13]$, mortality remains as high as $35 \%$, and disabling complications persist among ICU survivors, with recovery sometimes continuing for $>1$ year $[13,14]$. In addition, ARDS may be underrecognised and undertreated [14].

We hypothesised that patients with severe CAP and ARDS have a higher mortality than those patients who require mechanical ventilation without ARDS. The aim of the study was to assess the incidence, clinical characteristics, aetiology, risk factors and mortality of ICU patients with severe CAP and ARDS according to the new Berlin definition [12], compared with ventilated patients without ARDS.

\section{Methods}

\section{Study design and patients}

Refer to the online supplementary material for full details of the methods. This was a prospective observational cohort study of consecutive adult patients with CAP admitted to the ICU within $24 \mathrm{~h}$ of hospital admission, between November 1996 and December 2016. The inclusion criteria were as follows. Patients 1) met the criteria for severe CAP [15] and were admitted to the ICU, which included intermediate care units; and 2) received either IMV or noninvasive mechanical ventilation (NIMV) during the first $24 \mathrm{~h}$ of hospital admission. Patients were excluded if they had severe immunosuppression or active tuberculosis.

\section{Data collection and evaluation}

The following were recorded at admission: age, sex, smoking history, alcohol and drug consumption, comorbidities, antibiotic treatment in the 30 days before admission, treatment with oral and inhaled corticosteroids, clinical symptoms and signs, arterial blood gas measurements and chest radiography findings. All chest radiographs were reviewed by at least two co-authors, and consensus was required to define cases as ARDS or non-ARDS; in addition, we assessed laboratory parameters, diagnostic procedures, empiric antibiotic therapy, ventilator support (IMV and NIMV), pulmonary complications and other clinical events (cardiac arrhythmias, septic shock and acute renal failure). Duration of treatment, length of hospitalisation and mortality were noted. Finally, we calculated pneumonia severity index (PSI) [16] and the sepsis-related organ failure assessment (SOFA) [17] scores at hospital and ICU admission.

\section{Microbiological evaluation and diagnostic criteria}

We collected sputum samples, and when available, pleural fluid, tracheobronchial aspirates and bronchoalveolar lavage fluid. Sputum and blood samples were obtained for bacterial culture in the emergency department, before antibiotic therapy. Respiratory samples were processed for Gram and ZiehlNeelsen stains and for bacterial, fungal and mycobacterial cultures. Nasopharyngeal swabs for respiratory virus detection and urine samples for S. pneumoniae and Legionella pneumophila antigen detection were obtained within $24 \mathrm{~h}$ of hospital admission. Blood samples for serology of atypical pathogens and respiratory viruses were taken at admission and in the third and sixth weeks thereafter. The criteria for aetiological diagnosis can be found in a previous report [18].

\section{Definitions}

Pneumonia was defined as a new pulmonary infiltrate on chest radiography at hospital admission with symptoms and signs of lower respiratory tract infection. A prior episode of pneumonia was defined as a case within the past 12 months.

ARDS was identified within the first $24 \mathrm{~h}$ of hospital admission based on the Berlin definition [12]: new or worsening respiratory symptoms; bilateral pulmonary radiologic opacities, not fully explained by 
effusions; lobar/lung collapse or nodules, not fully explained by cardiac failure or fluid overload; and an arterial oxygen tension $\left(\mathrm{PaO}_{2}\right)$ /inspiratory oxygen fraction $\left(\mathrm{FIO}_{2}\right)$ ratio $\leqslant 300 \mathrm{mmHg}$ with a positive end-expiratory pressure or continuous positive airway pressure $>5 \mathrm{cmH}_{2} \mathrm{O}[12,19]$. The severity of ARDS was divided into three categories: mild $\left(200<\mathrm{PaO}_{2} / \mathrm{FIO}_{2} \leqslant 300 \mathrm{mmHg}\right)$, moderate $\left(100<\mathrm{PaO}_{2} / \mathrm{FIO}_{2} \leqslant 200 \mathrm{mmHg}\right)$ and severe $\left(\mathrm{PaO}_{2} / \mathrm{FIO}_{2}<100 \mathrm{mmHg}\right)$ [12]. We chose the initial $24 \mathrm{~h}$ to differentiate ARDS and non-ARDS patients because this period of time is considered the limit to differentiate between clinical features at admission and worsening evolution in the hospital [20]. Chronic obstructive pulmonary disease (COPD) was defined according to the 2017 Global Initiative for Chronic Obstructive Lung Disease strategy [21].

\section{Clinical outcomes}

The primary outcome was 30-day mortality. Other outcomes were length of hospitalisation and ICU and in-hospital mortality rates. Patients were followed for 30 days or until hospital discharge.

\section{Ethics statement}

The study was approved by the ethics committee of our institution (Hospital Clinic of Barcelona, Barcelona, Spain; no. 2009/5451). The need for written informed consent was waived due to the noninterventional design of the study.

\section{Statistical analysis}

Categorical variables are reported as $\mathrm{n}(\%)$, whereas continuous variables are reported as median (interquartile range) for non-normal distributions or as mean $\pm \mathrm{sD}$ for normal distributions. Categorical variables were compared using the Chi-squared test or the Fisher exact test, and continuous variables were compared using the t-test or the nonparametric Mann-Whitney U-test, as appropriate. The significance level was $\mathrm{p}<0.05$ (two-tailed), unless otherwise specified. All analyses were performed using IBM SPSS Statistics (version 23.0; IBM, Armonk, NY, USA).

Logistic regression analyses [22] were used to examine the associations between ARDS and risk factors. First, each risk factor was tested individually. Second, all risk factors with an association in the univariate model $(p<0.15)$ were added to the multivariate model. Finally, a backward stepwise selection $(\operatorname{pin}<0.05$, pout $<0.10$ ) was used to determine factors associated with ARDS. The association with 30 -day mortality was tested by univariate and multivariate analyses with the same inclusion criterion $(\mathrm{p}<0.15)$.

A propensity score was developed for patients with ARDS [23]. Together with the year of occurrence of pneumonia, the presence of ARDS, the microbial aetiology and the interaction between ARDS and the microbial aetiology, this was incorporated into the multivariate logistic regression analysis to predict 30 -day mortality. If two independent variables were highly correlated $(r>| \pm 0.30|)$, the variable with the largest variance was excluded from multivariate analysis [24]. The odds ratios (ORs) and 95\% confidence intervals were calculated.

The Hosmer-Lemeshow goodness-of-fit test was performed to assess the overall fit of the models. The areas under the receiver operating characteristic curves (AUCs) of the multivariate models were calculated to predict ARDS and 30-day mortality. Internal validation of the prediction models was conducted by ordinary nonparametric bootstrapping with 1000 bootstrap samples and bias-corrected, accelerated 95\% confidence intervals [25]. The same analyses were performed on the subset of patients with pneumococcal CAP. Finally, we used multiple imputation [26] to handle missing data.

\section{Results}

Patient characteristics

Among 5334 patients hospitalised with CAP during the observation period, 930 (17\%) were admitted to the ICU, of whom 462 (52\%) were not ventilated, 137 (15\%) received NIMV and 295 (33\%) received IMV (figure 1).

The study population comprised 432 patients treated in ICU with either IMV or NIMV: 125 cases (29\%) met the Berlin ARDS criteria, and 307 cases (71\%) did not. ARDS was present in 2\% of all patients hospitalised with CAP and in $13 \%$ of those admitted to the ICU. According to the severity classification based on the baseline $\mathrm{PaO}_{2} / \mathrm{FIO}_{2}$ ratio, 60 (48\%), 49 (40\%) and 15 (12\%) patients had mild, moderate and severe ARDS, respectively. Two patients had been ventilated in the prone position. None of them had received extracorporeal membrane oxygenation.

The patients' characteristics are shown in tables 1 and 2. Compared to patients without ARDS, those with ARDS had less often received treatment with inhaled corticosteroids; had less chronic respiratory comorbidity, particularly COPD; had poorer baseline oxygenation, higher organ dysfunction, and lower PSI risk; and had more multilobar involvement. Trends only were seen in less pneumococcal vaccination, fewer males and more frequent prior antibiotic treatment. 


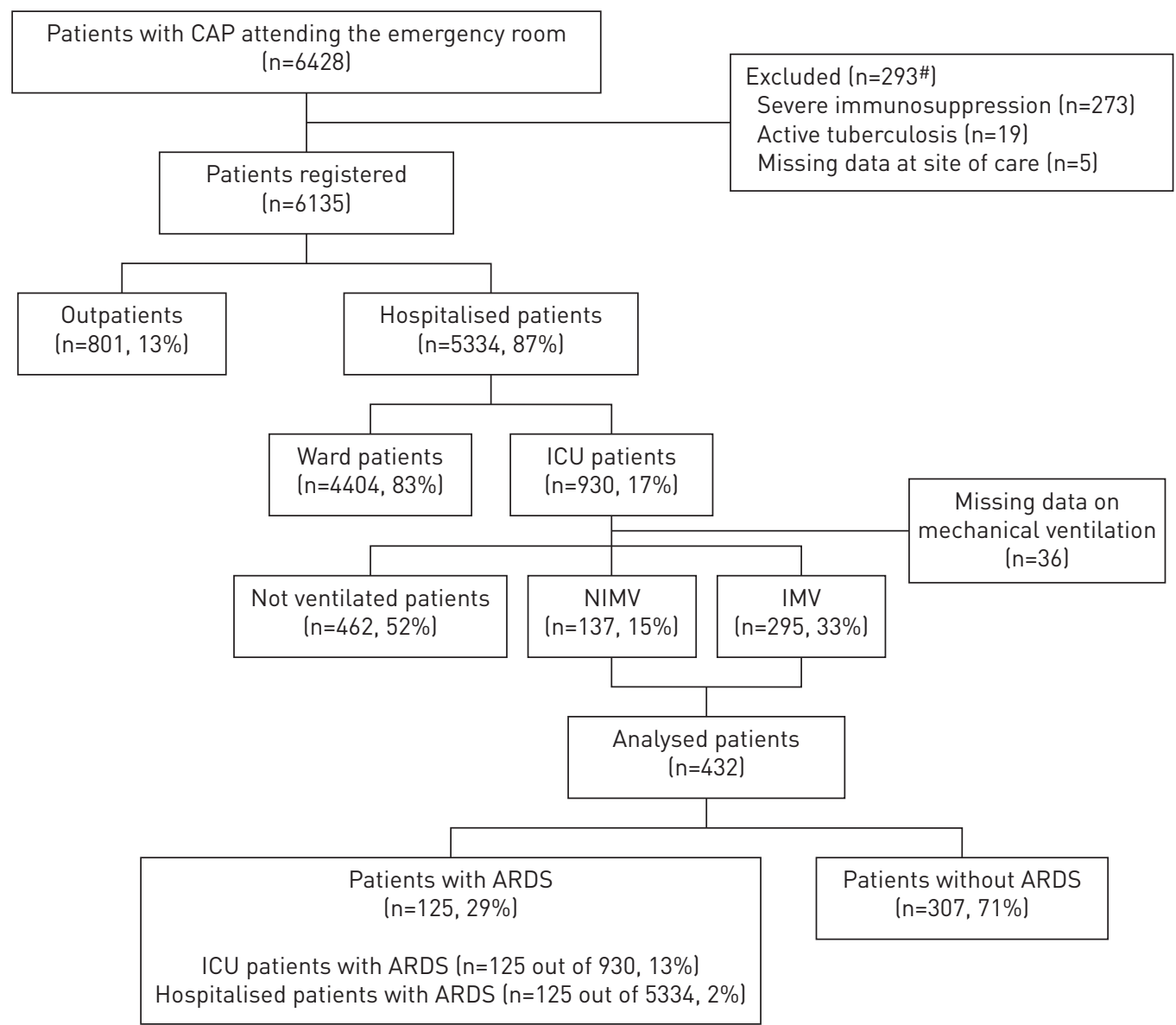

FIGURE 1 Flow diagram of the study population. CAP: community-acquired pneumonia; ICU: intensive care unit; NIMV: noninvasive mechanical ventilation; IMV: invasive mechanical ventilation; ARDS: acute respiratory distress syndrome. ${ }^{\#}$ : four patients had two exclusion criteria.

\section{Microbiological diagnosis}

An aetiological diagnosis was obtained in 228 (53\%) patients (table 3). The most frequent pathogen in both groups was $S$. pneumoniae, with no significant differences in aetiology between the groups. Among the 100 cases of pneumococcal CAP, 29 met the ARDS criteria, the same proportion as in the overall population.

\section{Empiric antibiotic therapy}

Data on empiric antibiotic treatment were available in 414 (96\%) patients (online supplementary table S1). The most frequent regimens were $\beta$-lactam plus either a respiratory fluoroquinolone $(42 \%)$ or a macrolide $(31 \%)$. ARDS patients more often received a $\beta$-lactam plus a respiratory fluoroquinolone compared with non-ARDS patients $(\mathrm{p}=0.003)$.

\section{Predictors of ARDS}

Among the variables associated with ARDS in the univariate analysis (table 4), a higher SOFA score and previous antibiotic treatment remained significant independent risk factors for ARDS in the multivariate analysis. Interestingly, prior treatment with inhaled corticosteroids was independently associated with a lower risk for ARDS. The AUC was 0.66 (95\% CI 0.60-0.71) for the model predictive of ARDS (online supplementary figure S1). Internal validation of the logistic regression model by bootstrapping demonstrated robust results for all the variables in the model, with small $95 \%$ confidence intervals around the original coefficients (online supplementary table S2).

\section{Mortality and length of stay}

We did not find significant differences between groups regarding mortality or length of hospitalisation (table 5). However, in cases with pneumococcal CAP, mortality in the ICU was higher among those with 


\begin{tabular}{|c|c|c|c|}
\hline & Non-ARDS patients & ARDS patients & p-value \\
\hline Subjects & 307 & 125 & \\
\hline Age years & $65.8 \pm 14.4$ & $63.1 \pm 17.3$ & 0.26 \\
\hline Male & 212 (69) & $75(60)$ & 0.071 \\
\hline Current smoker & $93(31)$ & $34 / 122(28)$ & 0.53 \\
\hline Current alcohol abuse & $66(22)$ & $34(28)$ & 0.21 \\
\hline Previous antibiotics ${ }^{\#}$ & $46(17)$ & $29(25)$ & 0.052 \\
\hline Influenza vaccine & 66 (38) & $26(34)$ & 0.62 \\
\hline Pneumococcal vaccine & $33(18)$ & 7 (9) & 0.054 \\
\hline Inhaled corticosteroids & $80(27)$ & $18(15)$ & 0.008 \\
\hline Systemic corticosteroids & $13(6)$ & $8(8)$ & 0.41 \\
\hline Previous episode of pneumonia & $29(11)$ & $7(6)$ & 0.18 \\
\hline Nursing home resident & $16(5)$ & $5(4)$ & 0.62 \\
\hline Comorbidity" & $244(81)$ & $83(66)$ & 0.002 \\
\hline Chronic respiratory disease & $152(51)$ & 45 (37) & 0.009 \\
\hline COPD & $105(36)$ & $26(22)$ & 0.005 \\
\hline Asthma & 9 (3) & $4(3)$ & 0.89 \\
\hline Bronchiectasis & $8(3)$ & $2(2)$ & 0.73 \\
\hline Other ${ }^{+}$ & $30(10)$ & $13(11)$ & 0.85 \\
\hline Chronic cardiovascular disease & $52(17)$ & $20(16)$ & 0.76 \\
\hline Diabetes mellitus & $75(25)$ & $26(21)$ & 0.38 \\
\hline Neurological disease & $46(16)$ & $20(17)$ & 0.84 \\
\hline Chronic renal disease & $27(9)$ & $8(6)$ & 0.37 \\
\hline Chronic liver disease & $26(9)$ & $12(10)$ & 0.74 \\
\hline
\end{tabular}

Data are presented as $n$, mean \pm SD or $n(\%)$, unless otherwise stated. ARDS: acute respiratory distress syndrome; COPD: chronic obstructive pulmonary disease. Percentages were calculated for nonmissing data. ": information on previous antibiotics was obtained in 67 (89\%) cases without any significant differences in the type of antibiotics each group had received: $\beta$-lactams $(n=38)$, fluoroquinolones $(n=12)$, macrolides ( $n=9)$ and other $(n=8) ;$ १: some patients may have more than one comorbid condition; ${ }^{+}$: other respiratory diseases included the sequelae of pulmonary tuberculosis, pulmonary hypertension and interstitial lung disease.

ARDS ( $\mathrm{p}=0.026$; online supplementary table S3), but neither the in-hospital mortality $(\mathrm{p}=0.070)$ nor the 30-day mortality $(\mathrm{p}=0.088)$ reached statistical significance.

According to ARDS severity, the 30 -day mortality rate was $32 \%, 33 \%$ and $60 \%$ for patients with mild, moderate and severe ARDS, respectively.

\section{Predictors of 30-day mortality}

Among the different variables associated with 30-day mortality in the univariate analysis (table 6), the following were independent predictors of 30-day mortality in the propensity-adjusted multivariate analysis: older age, previous antibiotic treatment, other chronic pulmonary diseases (including only the sequelae of pulmonary tuberculosis, pulmonary hypertension and interstitial lung disease), chronic cardiovascular and liver disease, higher SOFA score and inadequate empiric antibiotic treatment. It was notable that having a previous episode of pneumonia and having received the pneumococcal vaccination were independently associated to reduced 30 -day mortality rates.

Overall, ARDS was not associated with 30-day mortality, even after adjustment for potential confounders. The AUC was 0.79 (95\% CI $0.75-0.84)$ for the model predictive of 30-day mortality (online supplementary figure S2). Internal validation of the logistic regression model by bootstrapping demonstrated robust results for all variables included in the model, with small $95 \%$ confidence intervals around the original coefficients. Finally, the same analysis in the subset of patients with pneumococcal CAP confirmed that ARDS was not independently associated with 30-day mortality after adjustment for potential confounders (adjusted OR 1.77, 95\% CI 0.70-4.50; $\mathrm{p}=0.23$ ).

\section{Discussion}

The main findings of this study are as follows. First, based on the Berlin definition, ARDS was present in $2 \%$ of all hospitalised patients with CAP, $13 \%$ of those admitted to the ICU and $29 \%$ of those ICU patients requiring mechanical ventilatory support. Second, higher SOFA scores and previous antibiotic use 
TABLE 2 Patient characteristics at admission

\begin{tabular}{|c|c|c|c|}
\hline & Non-ARDS patients & ARDS patients & p-value \\
\hline Subjects & 307 & 125 & \\
\hline Mechanical ventilation & & & 0.55 \\
\hline Noninvasive & 100 (33) & $37(30)$ & \\
\hline Invasive & $207(67)$ & $88(70)$ & \\
\hline \multicolumn{4}{|l|}{ Laboratory findings } \\
\hline Creatinine $\mathrm{mg} \cdot \mathrm{dL}^{-1}$ & $1.3(1-1.8)$ & $1.3(0.9-2)$ & 0.52 \\
\hline C-reactive protein $\mathrm{mg} \cdot \mathrm{dL}^{-1}$ & $20.7(9.5-30)$ & $22.3(14.8-30.2)$ & 0.15 \\
\hline $\mathrm{PaO}_{2} / \mathrm{FlO}_{2}$ & $233(176-296)$ & $195(151-241)$ & $<0.001$ \\
\hline SOFA score $\#$ & $4(3-6)$ & $5(3-6)$ & 0.026 \\
\hline PSI score & $123(101-143)$ & 116 (81-139) & 0.017 \\
\hline PSI risk class ${ }^{\pi}$ & & & 0.020 \\
\hline I-III & 42 (19) & $29(31)$ & \\
\hline IV-V & $180(81)$ & $65(69)$ & \\
\hline Bacteraemia & $46(19)$ & 20 (19) & 0.98 \\
\hline Pleural effusion & $65(22)$ & 22 (18) & 0.37 \\
\hline Multilobar disease & $97(32)$ & $125(100)$ & $<0.001$ \\
\hline Septic shock & $106(36)$ & 45 (37) & 0.87 \\
\hline Acute renal failure & $140(47)$ & $61(50)$ & 0.63 \\
\hline \multicolumn{4}{|c|}{$\begin{array}{l}\text { Data are presented as } \mathrm{n}, \mathrm{n}(\%) \text { or median (interquartile range), unless otherwise stated. ARDS: acute } \\
\text { respiratory distress syndrome; } \mathrm{PaO}_{2} \text { : arterial oxygen tension; } \mathrm{FlO}_{2} \text { : inspiratory oxygen fraction; SOFA: } \\
\text { sepsis-related organ failure assessment; PSI: pneumonia severity index. Percentages were calculated on } \\
\text { nonmissing data. \#: higher SOFA score in ARDS patients was due to a higher scoring in the respiratory } \\
\text { component; } \overbrace{\text { : }} \text { stratified according to } 30 \text {-day risk mortality for community-acquired pneumonia: risk } \\
\text { classes I-III ( } \leqslant 90 \text { points) have low predicted mortality (range } 0-10 \% \text { ) and risk classes IV-V ( }>90 \text { points) } \\
\text { have the highest predicted mortality (range } 10-35 \% \text { ). }\end{array}$} \\
\hline
\end{tabular}

\begin{tabular}{|c|c|c|c|}
\hline & Non-ARDS patients & ARDS patients & p-value \\
\hline Subjects & 307 & 125 & \\
\hline Patients with defined aetiology & $157(51)$ & $71(57)$ & 0.29 \\
\hline Streptococcus pneumoniae & $71(45)$ & $29(41)$ & 0.54 \\
\hline Respiratory viruses & $12(8)$ & $8(11)$ & 0.37 \\
\hline Atypical & $10(6)$ & $5(7)$ & $>0.99$ \\
\hline Mycoplasma pneumoniae & $5(3)$ & $4(6)$ & 0.47 \\
\hline Coxiella burnetii & $1(1)$ & $1(1)$ & 0.53 \\
\hline Chlamydophila pneumoniae & 4 (3) & $0(0)$ & 0.31 \\
\hline Pseudomonas aeruginosa & $10(6)$ & $3(4)$ & 0.76 \\
\hline Staphylococcus aureus & $8(5)$ & $4(6)$ & $>0.99$ \\
\hline GNEB & $5(3)$ & $3(4)$ & 0.71 \\
\hline Escherichia coli & $3(2)$ & $3(4)$ & 0.38 \\
\hline Klebsiella pneumoniae & $2(1)$ & $0(0)$ & $>0.99$ \\
\hline Haemophilus influenzae & 5 (3) & 2 (3) & $>0.99$ \\
\hline Legionella pneumophila & $2(1)$ & $4(6)$ & 0.077 \\
\hline Streptococcus constellatus & $1(1)$ & $0(0)$ & $>0.99$ \\
\hline Moraxella catarrhalis & $1(1)$ & $0(0)$ & $>0.99$ \\
\hline Others & $2(1)$ & $0(0)$ & $>0.99$ \\
\hline Polymicrobial cases ${ }^{\#}$ & 30 (19) & $13(18)$ & 0.89 \\
\hline
\end{tabular}

Data are presented as $\mathrm{n}$ or $\mathrm{n}(\%)$, unless otherwise stated. ARDS: acute respiratory distress syndrome; GNEB: Gram-negative enteric bacilli. Percentages calculated on nonmissing data. The percentages of pathogens are related to the number of patients with aetiological diagnoses in each group. \#: polymicrobial cases: S. pneumoniae + respiratory viruses; S. pneumoniae + H. influenzae; S. pneumoniae + atypical bacteria; S. pneumoniae + E. coli; S. pneumoniae + S. aureus; S. pneumoniae + Acinetobacter spp.; S. pneumoniae + others; respiratory viruses + H. influenzae; respiratory viruses + S. aureus; respiratory viruses $+P$. aeruginosa; respiratory viruses + other; $P$. aeruginosa $+L$. pneumophila; $P$. aeruginosa $+K$. pneumoniae; $P$. aeruginosa + other; S. aureus + M. catarrhalis; S. aureus + atypical; S. aureus + P. aeruginosa. 
TABLE 4 Significant univariate and multivariate logistic regression analyses of acute respiratory distress syndrome (ARDS) predictors

\begin{tabular}{|c|c|c|c|c|}
\hline & \multicolumn{2}{|c|}{ Univariate ${ }^{\#}$} & \multicolumn{2}{|c|}{ Multivariate $\eta^{\eta+}$} \\
\hline & OR $(95 \% \mathrm{CI})$ & p-value & OR $(95 \% \mathrm{CI})$ & p-value \\
\hline Year of occurrence of pneumonia $\geqslant 2007$ & $0.96(0.64-1.46)$ & 0.86 & $0.99(0.64-1.54)$ & 0.98 \\
\hline Age +1 year & $0.99(0.98-1.00)$ & 0.12 & & \\
\hline Female & $1.49(0.97-2.29)$ & 0.071 & $1.52(0.97-2.39)$ & 0.067 \\
\hline Pneumococcal vaccination & $0.62(0.34-1.12)$ & 0.11 & & \\
\hline Inhaled corticosteroids & $0.45(0.26-0.77)$ & 0.004 & $0.48(0.27-0.85)$ & 0.012 \\
\hline Previous antibiotics & $1.60(0.99-2.61)$ & 0.057 & $1.80(1.08-2.98)$ & 0.024 \\
\hline Previous episode of pneumonia & $0.57(0.26-1.21)$ & 0.14 & & \\
\hline Chronic respiratory disease ${ }^{\S}$ & & 0.076 & & \\
\hline Nonchronic respiratory disease & 1 & & & \\
\hline COPD & $0.47(0.28-0.79)$ & 0.004 & & \\
\hline Asthma & $0.72(0.27-1.92)$ & 0.51 & & \\
\hline Bronchiectasis & $0.96(0.28-3.29)$ & 0.95 & & \\
\hline Other ${ }^{f}$ & $0.81(0.40-1.63)$ & 0.55 & & \\
\hline SOFA score $(+1 \text { point })^{\# \#}$ & $1.12(1.03-1.22)$ & 0.007 & $1.13(1.03-1.23)$ & 0.009 \\
\hline Microbial aetiology & & 0.36 & & 0.37 \\
\hline Unknown & 1 & & & \\
\hline Streptococcus pneumoniae & $1.10(0.67-1.82)$ & 0.70 & $1.17(0.69-1.97)$ & 0.56 \\
\hline Other & $1.45(0.87-2.41)$ & 0.15 & $1.46(0.86-2.47)$ & 0.16 \\
\hline \multicolumn{5}{|c|}{ 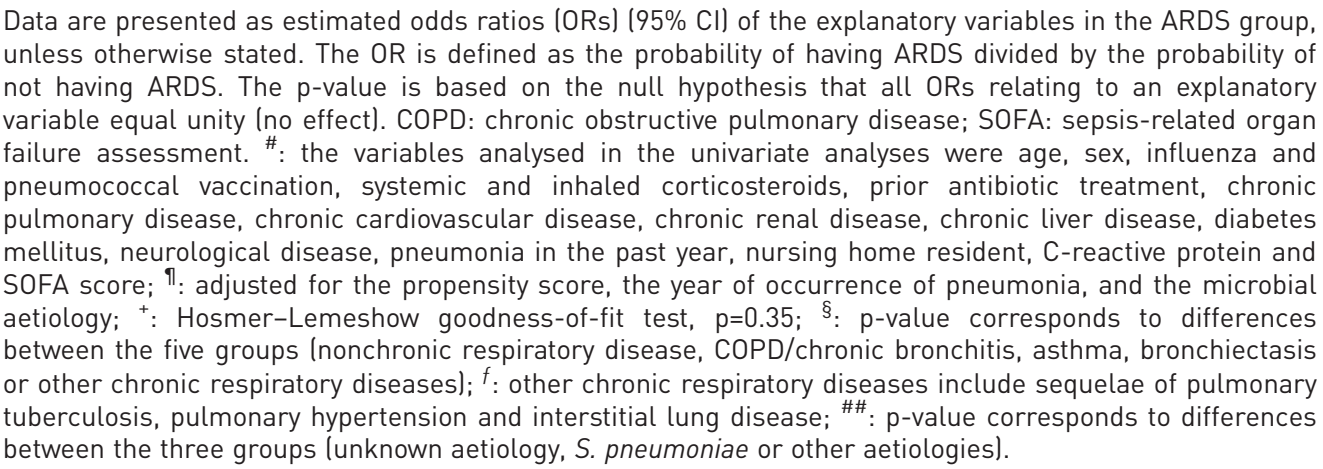 } \\
\hline
\end{tabular}

were independent predictors for ARDS among ventilated patients with CAP, while previous inhaled corticosteroid treatment was protective. Third, ARDS had no difference in aetiologies or mortality in patients requiring mechanical ventilation for CAP.

ARDS is recognised globally as a major clinical problem [13], with pneumonia and extrapulmonary sepsis being the main risk factors in $75 \%$ of cases [14]. However, there has been a lack of information about the incidence of ARDS in CAP since the Berlin definition was proposed. Our data are consistent with those of LUNG SAFE (Large Observational Study to Understand the Global Impact of Severe Acute Respiratory Failure) [14], which evaluated the incidence of ARDS in 459 ICUs in 50 countries. They reported that

\begin{tabular}{lccc} 
TABLE 5 Clinical outcomes & & \\
& Non-ARDS patients & ARDS patients & p-value \\
\hline Subjects & 307 & 125 & \\
Length of hospital stay days & $15(10-27)$ & $16(9-30)$ & 0.96 \\
ICU mortality & $70(23)$ & $37(30)$ & 0.14 \\
In-hospital mortality & $81(26)$ & $41(33)$ & 0.18 \\
30-day mortality & $90(30)$ & $44(35)$ & 0.25
\end{tabular}

Data are presented as $n$, median (interquartile range) or $n(\%)$, unless otherwise stated. ARDS: acute respiratory distress syndrome; ICU: intensive care unit. Percentages were calculated for nonmissing data. 
TABLE 6 Significant univariate and multivariate logistic regression analyses for predictors of 30-day mortality

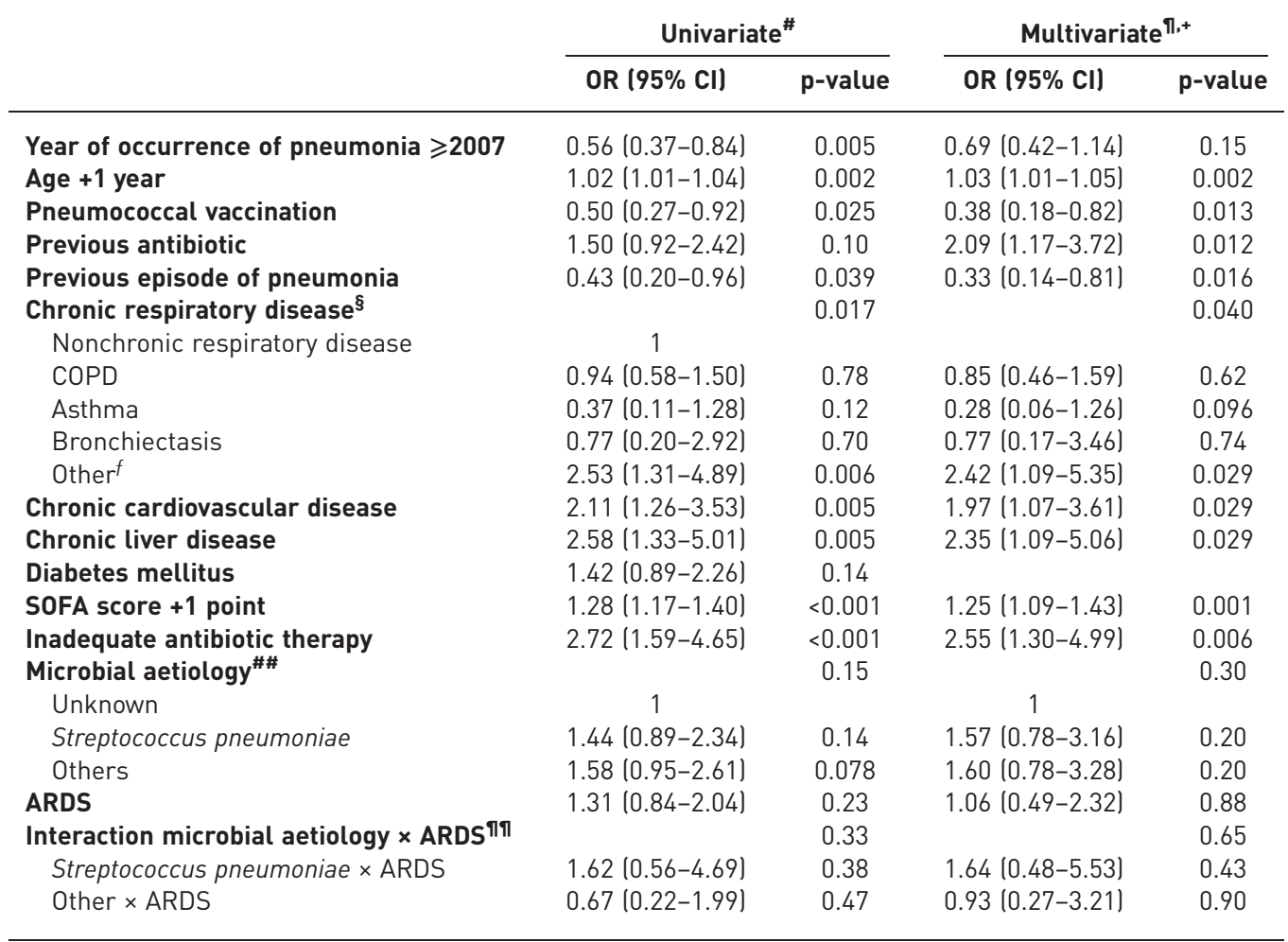

Data are shown as estimated odds ratios (ORs) $(95 \% \mathrm{Cls}$ ) of the explanatory variables in the 30 -day mortality group. The OR is defined as the probability of 30 -day mortality divided by the probability of no 30-day mortality. The $\mathrm{p}$-value is based on the null hypothesis that all ORs relating to an explanatory variable equal unity (no effect). COPD: chronic obstructive pulmonary disease; SOFA: sepsis-related organ failure assessment; ARDS: acute respiratory distress syndrome. " : the variables analysed in the univariate analyses were age, sex, influenza and pneumococcal vaccination, systemic and inhaled corticosteroids, prior antibiotic treatment, chronic pulmonary disease, chronic cardiovascular disease, chronic renal disease, chronic liver disease, diabetes mellitus, neurological disease, pneumonia in the past year, nursing home resident, creatinine, C-reactive protein, SOFA score and inadequate antibiotic therapy; ": adjusted for propensity score, year of occurrence of pneumonia, ARDS, microbial aetiology and the interaction between ARDS and microbial aetiology; ${ }^{+}$: Hosmer-Lemeshow goodness-of-fit test, $p=0.27 ;{ }^{\S}$ : $p$-value corresponds to differences between the five groups (nonchronic respiratory disease, COPD/chronic bronchitis, asthma, bronchiectasis or other chronic respiratory diseases); $f$ : other chronic respiratory diseases include sequelae of pulmonary tuberculosis, pulmonary hypertension and interstitial lung disease; \#\#: p-value corresponds to differences between the three groups lunknown aetiology, S. pneumoniae or other aetiologies); ๆศा: p-value corresponds to the interaction between microbial aetiology and ARDS.

ARDS occurred in $10 \%$ of all ICU admissions and in $23 \%$ patients who required mechanical ventilation. According to the Berlin criteria, 30\%, $47 \%$ and $23 \%$ of their patients had mild, moderate and severe ARDS, respectively. In contrast, there was a higher proportion of mild ARDS and a lower proportion of severe ARDS in our cohort.

Over the past century only a few small case series of ARDS in pneumococcal CAP have been reported [27-29]. A recent French multicentre study [30] concerning pneumococcal CAP in the ICU indicated that the incidence of ARDS was $45 \%$, which was substantially higher than that reported in this study. However, those authors used the prior American-European consensus definition of ARDS [31] and not the current Berlin definition, and included nonventilated patients (16\%).

To our knowledge, this is the first study to provide data on the incidence of ARDS using the Berlin definition among patients hospitalised with CAP. We studied a cohort with severe CAP requiring mechanical ventilation, and compared those with and without ARDS. Regarding the independent predictors for ARDS, the association of higher organ system dysfunction to ARDS was to be expected because these patients had worse baseline oxygenation, an important component of the score. 
We previously reported that inhaled corticosteroid use before admission in patients hospitalised because of CAP can reduce the systemic inflammatory response [32], possibly with a selective modulation of the mechanisms of defences to infection [33], with reduced serum levels of tumour necrosis factor- $\alpha$, among others. This biomarker has been involved in the pathophysiology of ARDS [34]

We have no clear explanation for the independent association between previous antibiotic use and increased risk of ARDS. To our knowledge, this association has not previously been reported. We have shown that previous antibiotic use can be associated with antibiotic-resistant bacteraemia in patients with CAP [35], resulting in further inappropriate therapy. Whether this could predispose patients to ARDS remains to be assessed. However, this association requires confirmation in future studies.

Mortality among ARDS patients was no different to that among ventilated non-ARDS patients. Indeed, even the propensity-adjusted multivariate analysis did not show an association between ARDS and patient mortality. According to our results, we think that the expected association of ARDS with mortality seems more related to the need for mechanical ventilation in these patients with CAP rather than ARDS itself, as we have recently reported that invasive mechanical ventilation in patients with severe CAP independently predicts mortality [36]. However, we cannot exclude that specific populations of patients with CAP and ARDS may have different mortality, since subphenotypes of ARDS patients with different outcomes related to inflammation [37] or fluid responsiveness [38] have been proposed.

Independent predictors of mortality have previously been reported for CAP and other pulmonary infections in critically-ill patients, including older age, chronic liver disease [39], chronic cardiovascular disease [39-41], increased organ system dysfunction [42-44] and inadequate empiric treatment [45]. The reason for the association of previous pneumonia with lower mortality is unclear; possibly these patients might have developed a more effective immune response to infection. This is consistent with pneumococcal vaccination being independently associated with reduced mortality. Given that ARDS was related to mortality in patients with pneumococcal pneumonia, previous vaccination might reduce disease severity in these patients.

Due to the association of ARDS with higher mortality in pneumococcal pneumonia, there is a need to develop effective preventive measures for this complication. A randomised clinical trial [46] and a meta-analysis [47] found that short-term treatment with corticosteroids can prevent radiographic progression and ARDS development in CAP. However, these studies investigated CAP in general, not pneumococcal pneumonia specifically. Studies focusing on the acute treatment of pneumococcal pneumonia with corticosteroids or other immunomodulators [48] are therefore needed. Unfortunately, we have no systematic data on the number of patients who received pneumococcal conjugate vaccine-13 or pneumococcal polysaccharide vaccine- 23 .

In our opinion, the strengths of this study are the large sample, the prospective and consecutive data collection, the use of the current Berlin definition for ARDS and the statistical analysis by propensity scoring to account for bias due to observed confounders. We think this study provides reliable data on the incidence of ARDS in patients with severe CAP, and that these data can be used for future studies in this important population. In addition, we assessed the association of ARDS with mortality in mechanically ventilated patients to avoid potential bias due to worse outcomes associated with the need for ventilatory support.

Some limitations need to be addressed. First, the long period of recruitment (20 years) has undoubtedly been associated with major advances in patient care. Although we did incorporate advances in ventilatory management [10] and other support measures [13] over this time, our management protocol for CAP did not change substantially. We allowed for this by including the period of admission when adjusting mortality outcomes. Second, this study was conducted at a single centre, which necessitates cautious extrapolation of the findings to other settings. Third, the Berlin definition requires the use of positive airway pressure, which is only applied to patients receiving ventilatory support. Fourth, the rate of respiratory viruses identified in our population may be underestimated since the diagnostic techniques employed were routinely implemented over the recruitment period.

In conclusion, ARDS complicating severe CAP occurs in $29 \%$ of ventilated patients, but is unrelated to either the aetiology or mortality. ARDS criteria should not be considered in the choice of the empiric antibiotic treatment in these patients.

Acknowledgements: We are indebted to the nursing staff and the attending physicians for their cooperation in this study.

Author contributions: A. Torres and M. Ferrer are the guarantors of the entire manuscript, being responsible for all content, including the collected data and its analysis. 
Conflict of interest: None declared.

Support statement: This work was supported by Ciber de Enfermedades Respiratorias (CibeRes CB06/06/0028), 2009 Support to Research Groups of Catalonia 911, and IDIBAPS (CERCA Programme/Generalitat de Catalunya). C. Cilloniz is in receipt of an ERS Short Term Fellowship and Postdoctoral Grant ("Strategic plan for research and innovation in health-PERIS 2016-2020"). A. Ceccato is in receipt of an SEPAR-ALAT Fellowship. Funding information for this article has been deposited with the Crossref Funder Registry.

\section{References}

1 File TM Jr, Marrie TJ. Burden of community-acquired pneumonia in North American adults. Postgrad Med 2010; 122: $130-141$.

2 Ewig S, Torres A. Community-acquired pneumonia as an emergency: time for an aggressive intervention to lower mortality. Eur Respir J 2011; 38: 253-260.

3 Leoni D, Rello J. Severe community-acquired pneumonia: optimal management. Curr Opin Infect Dis 2017; 30: 240-247.

4 Liapikou A, Ferrer M, Polverino E, et al. Severe community-acquired pneumonia: validation of the Infectious Diseases Society of America/American Thoracic Society guidelines to predict an intensive care unit admission. Clin Infect Dis 2009; 48: 377-385.

5 Lenz H, Norby GO, Dahl V, et al. Five-year mortality in patients treated for severe community-acquired pneumonia - a retrospective study. Acta Anaesthesiol Scand 2017; 61: 418-426.

6 Woodhead M, Welch CA, Harrison DA, et al. Community-acquired pneumonia on the intensive care unit: secondary analysis of 17,869 cases in the ICNARC Case Mix Programme Database. Crit Care 2006; 10: Suppl. 2, S1.

7 Cillóniz C, Ewig S, Polverino E, et al. Microbial aetiology of community-acquired pneumonia and its relation to severity. Thorax 2011; 66: 340-346.

8 Cillóniz C, Ewig S, Polverino E, et al. Pulmonary complications of pneumococcal community-acquired pneumonia: incidence, predictors, and outcomes. Clin Microbiol Infect 2012; 18: 1132-1142.

9 Ceccato A, Torres A, Cilloniz C, et al. Invasive disease vs urinary antigen-confirmed pneumococcal community-acquired pneumonia. Chest 2017; 151: 1311-1319.

10 Fan E, Del Sorbo L, Goligher EC, et al. An official American Thoracic Society/European Society of Intensive Care Medicine/Society of Critical Care Medicine clinical practice guideline: mechanical ventilation in adult patients with acute respiratory distress syndrome. Am J Respir Crit Care Med 2017; 195: 1253-1263.

11 Thompson BT, Chambers RC, Liu KD. Acute respiratory distress syndrome. N Engl J Med 2017; 377: 562-572.

12 Ranieri VM, Rubenfeld GD, Thompson BT, et al. Acute respiratory distress syndrome: the Berlin Definition. JAMA 2012; 307: 2526-2533.

13 Confalonieri M, Salton F, Fabiano F. Acute respiratory distress syndrome. Eur Respir Rev 2017; 26: 160116.

14 Bellani G, Laffey JG, Pham T, et al. Epidemiology, patterns of care, and mortality for patients with acute respiratory distress syndrome in intensive care units in 50 countries. JAMA 2016; 315: 788-800.

15 Mandell LA, Wunderink RG, Anzueto A, et al. Infectious Diseases Society of America/American Thoracic Society consensus guidelines on the management of community-acquired pneumonia in adults. Clin Infect Dis 2007; 44: Suppl. 2, S27-S72.

16 Fine MJ, Auble TE, Yealy DM, et al. A prediction rule to identify low-risk patients with community-acquired pneumonia. $N$ Engl J Med 1997; 336: 243-250.

17 Vincent JL, Moreno R, Takala J, et al. The SOFA (sepsis-related organ failure assessment) score to describe organ dysfunction/failure. On behalf of the Working Group on Sepsis-Related Problems of the European Society of Intensive Care Medicine. Intensive Care Med 1996; 22: 707-710.

18 Cillóniz C, Polverino E, Ewig S, et al. Impact of age and comorbidity on cause and outcome in community-acquired pneumonia. Chest 2013; 144: 999-1007.

19 Ferguson ND, Fan E, Camporota L, et al. The Berlin definition of ARDS: an expanded rationale, justification, and supplementary material. Intensive Care Med 2012; 38: 1573-1582.

20 Restrepo MI, Mortensen EM, Rello J, et al. Late admission to the ICU in patients with community-acquired pneumonia is associated with higher mortality. Chest 2010; 137: 552-557.

21 Vogelmeier CF, Criner GJ, Martinez FJ, et al. Global Strategy for the Diagnosis, Management, and Prevention of Chronic Obstructive Lung Disease 2017 Report. GOLD Executive Summary. Am J Respir Crit Care Med 2017; 195: 557-582.

22 Hosmer DW, Lemeshow S, eds. Applied Logistic Regression. 2nd Edn. New York, John Wiley \& Sons, 2000; chapters 2-5.

23 Joffe MM, Rosenbaum PR. Invited commentary: propensity scores. Am J Epidemiol 1999; 150: 327-333.

24 Healey JF. Statistics: a Tool for Social Research. Belmont, CA, Wadsworth Publishing Company, 2011.

25 Efron B, Tibshirani R. An Introduction to the Bootstrap (Monographs on Statistics and Applied Probability. New York, Chapman and Hall, 1993.

26 Steyerberg E. Clinical Prediction Models. A Practical Approach to Development, Validation, and Updating. New York, Springer, 2009.

27 Fruchtman SM, Gombert ME, Lyons HA. Adult respiratory distress syndrome as a cause of death in pneumococcal pneumonia. Report of ten cases. Chest 1983; 83: 598-601.

28 Torres A, Agusti A, Rodriguez-Roisin R, et al. ARDS and pneumococcal pneumonia. Chest 1984; 85: 584.

29 Mannes GP, Boersma WG, Baur $\mathrm{CH}$, et al. Adult respiratory distress syndrome (ARDS) due to bacteraemic pneumococcal pneumonia. Eur Respir J 1991; 4: 503-504.

30 Mongardon N, Max A, Bouglé A, et al. Epidemiology and outcome of severe pneumococcal pneumonia admitted to intensive care unit: a multicenter study. Crit Care 2012; 16: R155.

31 Bernard GR, Artigas A, Brigham KL, et al. The American-European Consensus Conference on ARDS. Definitions, mechanisms, relevant outcomes, and clinical trial coordination. Am J Respir Crit Care Med 1994; 149: 818-824.

32 Ferrer M, Torres A, Martínez R, et al. Inhaled corticosteroids and systemic inflammatory response in community-acquired pneumonia: a prospective clinical study. Respirology 2014; 19: 929-935. 
33 Gutierrez P, Closa D, Piñer R, et al. Macrophage activation in exacerbated COPD with and without community-acquired pneumonia. Eur Respir J 2010; 36: 285-291.

34 Butt Y, Kurdowska A, Allen TC. Acute lung injury: a clinical and molecular review. Arch Pathol Lab Med 2016; 140: 345-350.

35 Torres A, Cillóniz C, Ferrer M, et al. Bacteraemia and antibiotic-resistant pathogens in community acquired pneumonia: risk and prognosis. Eur Respir J 2015; 45: 1353-1363.

36 Ferrer M, Travierso C, Cilloniz C, et al. Severe community-acquired pneumonia: characteristics and prognostic factors in ventilated and non-ventilated patients. PLoS One 2018; 13: e0191721.

37 Bos LD, Schouten LR, van Vught LA, et al. Identification and validation of distinct biological phenotypes in patients with acute respiratory distress syndrome by cluster analysis. Thorax 2017; 72: 876-883.

38 Famous KR, Delucchi K, Ware LB, et al. Acute respiratory distress syndrome subphenotypes respond differently to randomized fluid management strategy. Am J Respir Crit Care Med 2017; 195: 331-338.

39 Di Pasquale M, Esperatti M, Crisafulli E, et al. Impact of chronic liver disease in intensive care unit acquired pneumonia: a prospective study. Intensive Care Med 2013; 39: 1776-1784.

40 Ranzani OT, Ferrer M, Esperatti M, et al. Association between systemic corticosteroids and outcomes of intensive care unit-acquired pneumonia. Crit Care Med 2012; 40: 2552-2561.

41 Martin-Loeches I, Torres A, Rinaudo M, et al. Resistance patterns and outcomes in intensive care unit (ICU)-acquired pneumonia. Validation of European Centre for Disease Prevention and Control (ECDC) and the Centers for Disease Control and Prevention (CDC) classification of multidrug resistant organisms. J Infect 2015; 70: 213-222.

42 Tejerina E, Frutos-Vivar F, Restrepo MI, et al. Prognosis factors and outcome of community-acquired pneumonia needing mechanical ventilation. J Crit Care 2005; 20: 230-238.

43 Pascual FE, Matthay MA, Bacchetti P, et al. Assessment of prognosis in patients with community-acquired pneumonia who require mechanical ventilation. Chest 2000; 117: 503-512.

44 Lee JH, Ryu YJ, Chun EM, et al. Outcomes and prognostic factors for severe community-acquired pneumonia that requires mechanical ventilation. Korean J Intern Med 2007; 22: 157-163.

45 Alvarez-Lerma F. Modification of empiric antibiotic treatment in patients with pneumonia acquired in the intensive care unit. ICU-acquired Pneumonia Study Group. Intensive Care Med 1996; 22: 387-394.

46 Torres A, Sibila O, Ferrer M, et al. Effect of corticosteroids on treatment failure among hospitalized patients with severe community-acquired pneumonia and high inflammatory response: a randomized clinical trial. JAMA 2015; 313: 677-686.

47 Wan YD, Sun TW, Liu ZQ, et al. Efficacy and safety of corticosteroids for community-acquired pneumonia: a systematic review and meta-analysis. Chest 2016; 149: 209-219.

48 Welte $\mathrm{T}$, Dellinger RP, Ebelt $\mathrm{H}$, et al. Concept for a study design in patients with severe community-acquired pneumonia: a randomised controlled trial with a novel IGM-enriched immunoglobulin preparation - the CIGMA study. Respir Med 2015; 109: 758-767. 